\title{
Study on the Hierarchical Structure of the "Belt and Road" Aviation Network Based on K-Core Analysis
}

\author{
Hongguang Yao $\mathbb{D}$, Huihui Xiao $\mathbb{D}$, and Wei Wei \\ College of Air Transportation, Shanghai University of Engineering Science, Shanghai 201620, China \\ Correspondence should be addressed to Hongguang Yao; yhg1yhg@sina.com
}

Received 23 September 2021; Accepted 9 December 2021; Published 8 February 2022

Academic Editor: Akbar Ali

Copyright (c) 2022 Hongguang Yao et al. This is an open access article distributed under the Creative Commons Attribution License, which permits unrestricted use, distribution, and reproduction in any medium, provided the original work is properly cited.

\begin{abstract}
Since the "Belt and Road" Initiative, air transport has become the second largest mode of transport after ocean shipping and has contributed more and more to international trade between the countries. To explore the hierarchical characteristics of the aviation network of the "Belt and Road," analyze the relationship between levels, and identify the core layer of the network, $k$-core analysis based on the "degree" value was designed and performed; the data of airports and air routes were collected to construct the "Belt and Road" aviation network model; then, $k$-core decomposition was conducted to reduce the size of nodes layer by layer from the outside to the inside, and a network structure model with 19 levels was obtained; the relationship between the coreness and centrality of nodes in the network was investigated, and the changes in the attribute of networks at different levels and the connection between networks were explored; according to the structural characteristics of the network, the "Belt and Road" aviation network was divided into three categories: the core layer, the middle layer, and the detail layer. The results showed that the highest-level network obtained by $k$-core decomposition was used as the core layer of the "Belt and Road" aviation network, including 53 airports in 37 countries, all of which have high degree centrality and eigenvector centrality; the core layer network exhibits strong "small world" characteristics; there is little difference between the degree values of nodes, and the network has high stability. The status of China's airports in the core layer network is not prominent; the four regional sectors such as East Asia, Southeast Asia, Central Asia, and the Middle East are closely connected regarding air transport. Europe is relatively weakly connected with three sectors: East Asia, Southeast Asia, and Central Asia; geographical factors are still the dominant factors determining the status of hub airports along the "Belt and Road."
\end{abstract}

\section{Introduction}

Since 2013, air transportation has become the second largest mode of transport after ocean shipping. Compared with ocean shipping, air transportation is less limited by geographical environment, more timely and more accessible. In addition, the influence of geopolitical and security situation is also significantly less than that of ocean shipping. With its unique technical and economic advantages, air transportation has maintained a rapid growth momentum in recent years. Taking China as an example, air transport is growing by more than $20 \%$ per year between China and the other countries of the "Belt and Road" before the global spread of COVID-19. In 2019, air transport accounted for 26 percent of China's total imports and exports. Among 167 international routes opened in 2019, 105 or 62.87 percent was accessible to countries along the "Belt and Road." The aviation network between countries along the "Belt and Road" is becoming more and more perfect, contributing more and more to international trade. However, the aviation network of the "Belt and Road" is a complex network consisting of more than 1,000 general airports and tens of thousands of air routes; a few key airports play a dominant role in the service function and service ability of the whole aviation network. Therefore, identifying the core layer that plays a leading role through the hierarchical analysis of the aviation network is the key to further optimize the aviation network structure and provide a stronger support for the "Belt and Road" Initiative. 
At present, abundant results have been achieved in the research of the characteristics of aviation network structure. Empirical research on the network topology revealed that the international air transport network is "scale-free" with "small world" characteristics [1]; study on the degree centrality, closeness centrality, and eigenvector topological centrality of airports in the aviation network showed that North America and East Asia focus on the development of regional hub airports while Western Europe, Southeast Asia, and the Middle East focus on building their main airports into global hubs [2]; although China's aviation network also has the characteristics of "small world" and "scale-free" [3], it is different from the global aviation network in which the difference in nodes is more prominent [4]. Besides, the aviation network of the "Belt and Road" has also become a research focus. Plenty of scholars have explored the spatial pattern and evolution of the aviation network of the "Belt and Road" through empirical research and pointed out the unbalanced overall spatial distribution of the aviation network of the "Belt and Road" $[5,6]$. The heterogeneity of nodes is a remarkable feature of aviation network [7]. A large number of scholars adopted centrality analysis to study the differences between nodes in the network. They calculated the "degree centrality, betweenness centrality, and closeness centrality" of nodes to study the status of different nodes in the network and investigated their driving factors [8-10]. The abovementioned methods can find the airports playing a central role in the network but fail to identify the core layer of the aviation network, making the research results difficult to be used to further optimize the aviation network structure. Therefore, many scholars have tried to study the core groups in the network through the cohesive subgroups analysis [11, 12], analyzed the cliques in the network and the relationship within and between cliques by such methods as cliques, $n$-clique, $k$-cluster, and $k$-core [13], and explored the "substructures" of the network [14]; a series of algorithms were used to reveal how the structure of the network is composed of substructures [15] and how some important substructures exert direct impact on the overall function of the network [16]. Therefore, using $k$-core analysis based on the "degree" value to study the hierarchical structure of the aviation network and identifying the core layer of the network by analyzing the relationship between the layers can provide theoretical guidance for the further improvement of the "Belt and Road" aviation network.

\section{The $k$-Core Analysis of the Network}

2.1. The $k$-Core of the Network. Seidman proposed the $k$-core analysis method in 1983, believing that the minimum degree can be used to determine the groups with different cohesiveness in the network and realize the research on the hierarchical structure of the network [17]. $k$-core is a concept of subgroups based on degree value; if the network is abstracted as a graph $G=(V, E)$ composed of a point set $V$ and an edge set $E$, then $k$-core refers to a subgraph that satisfies the following condition; that is, each node in the subgraph is at least connected with $k$ nodes in this subgraph [18]. The $k$ core of the network can be obtained by recursively removing all nodes with a degree value less than $k$ and their edges until all nodes in the residual network have a degree value of at least $k$. The coreness of a node represents the deepest $k$-core that contains the node, and the maximum value of the coreness of nodes is defined as the coreness of the network. The set of nodes that exists in the $k$-core and not in the $(k+1)$-core is defined as the " $k$-residual set" [19].

2.2. $k$-Core Decomposition. The $k$-core decomposition can reveal the hierarchical characteristics of the network [20]. $K$-core decomposition is realized by recursively removing all nodes with a degree value less than or equal to $K$ in the network; since nodes with small degree values are generally located in the outer layer of the network and nodes with large degree values are often located in the inner layer of the network, the $k$-core decomposition is conducted layer by layer from the outside to the inside [21].

The process of $k$-core decomposition is described in Figure 1; in a specific network, the first degree value of each node in the network is calculated; then, according to the order of the degree values from small to large, the corresponding nodes and edges in the network are removed in turn. As shown in Figure 1, three nodes such as $v 19, v 18$, and $v 22$ are isolated points with a degree value of 0 . Therefore, these three nodes are removed firstly. The remaining nodes and their edges form a new subgraph, namely, 1-core; then, the nodes with a degree value of 1 in the network and their corresponding edges are removed $(v 11, v 16, v 17, v 18, v 21$, $v 23)$ to obtain 2-core; the nodes with a degree value of 2 $(v 3, v 4, v 5, v 13)$ and their corresponding edges in the network are removed to obtain 3-core; after removing the nodes with a degree value of 3 and their corresponding edges, all nodes and edges do not exist, and the decomposition process is terminated; the subgraph corresponding to the 3-core can be regarded as the core layer of the network.

In the process of $k$-core decomposition, the nodes are removed together with their corresponding edges. Therefore, although some nodes have different initial degree values during decomposition, they may eventually be kept at the same level. For example, the initial degree value of the $v 3$ node is 3 , but when the $v 16$ node and its corresponding edge are removed, the degree value of $v 3$ is 2 ; thus, it is kept in the 2-core.

\section{3. $k$-Core Analysis of the "Belt and Road" Aviation Network}

3.1. Data Collection and Network Construction. In this paper, an aviation network model was established using the airports with international air routes in 65 countries along the "Belt and Road" as nodes and the air routes among these countries as edges. A total of 522 airports and 6,822 international air routes were collected. The data come from the website (http://www.variflight.com). With 522 airports as the rows and columns of the matrix, the $0-1$ adjacency matrix A of the "Belt and Road" aviation network was constructed based on whether there are air routes between countries. 


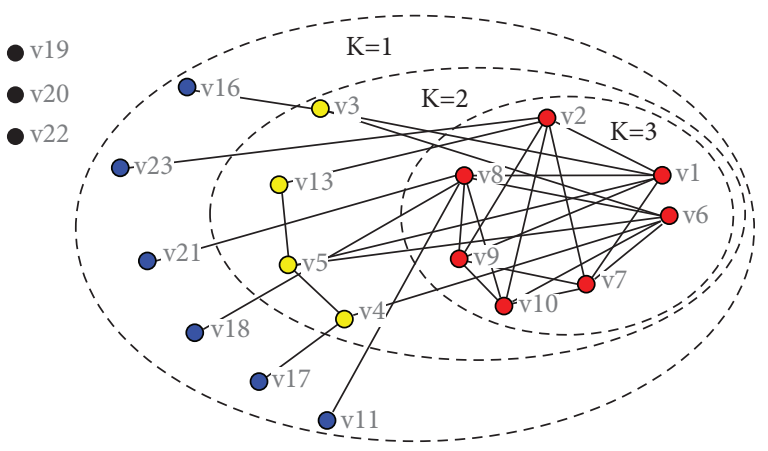

Figure 1: $k$-core decomposition.

3.2. $k$-Core Decomposition of the Aviation Network of "Belt and Road". The adjacency matrix A was input into the UCINET software for $k$-core analysis so that the coreness of airports in the aviation network can be calculated; the calculation shows that the maximum coreness is 19 . As the coreness $k$ increases, the extinction of nodes and edges in each layer of the network is shown in Table 1 .

It can be seen from Table 1 that when $k=2$ and $k=3$, the change in node is the greatest, indicating that there are highdensity subgroups in these two levels; when $k=4$ and $k=5$, the remaining nodes drop to $8 \%$, and the subsequent sequence maintains a relatively stable state, suggesting that in the aviation network of "Belt and Road," high-density subgroups of nodes are mainly distributed on the periphery of the network, which are manifested as the state-level and regional aviation network subsystems; when $k>6$, the extinction of nodes is relatively stable, indicating that the highdensity subgroups have disappeared. The subsequent network hierarchy is balanced, and the main structure of the network appears layer by layer. Generally, the extinction of edges is relatively stable, slightly increasing with the value of $k$, demonstrating that the peripheral nodes have a relatively small number of air routes.

\subsection{Analysis of the Correlation between the Coreness and Centrality of the Nodes in "Belt and Road" Aviation Network}

3.3.1. Centrality Indicators of Node. According to different standards, the centrality of nodes can be divided into four types: degree centrality, betweenness centrality, closeness centrality, and eigenvector centrality.

(1) Degree Centrality. If a node is directly connected to many other nodes, then it is located at a relatively central position [22]. Relative degree centrality is shown in equation (1), where $n$ is the number of nodes in the network and $d_{x}$ is the degree of node $x$.

$$
C^{\prime} \mathrm{RD}_{(x)}=\frac{d_{x}}{n-1} \text {. }
$$

(2) Betweenness Centrality. If a node is on many lines, then it has the ability to control the connection of other nodes, thereby further affecting the function of the entire network [23]. Relative betweenness centrality is defined in the following equation:

$C_{\mathrm{ABk}}=\frac{2\left(\sum_{i}^{n} \sum_{j}^{n} g_{i j}(d) / g_{i j}\right)}{n^{2}-3 n+2}, \quad i \neq j \neq d, i<j$,

where $g_{i j}$ represents the number of shortest paths between node $i$ and $j$ and $g_{i j}(d)$ is the number of shortest paths between node $i$ and $j$ passing through node $d$.

(3) Closeness Centrality. The closer the node is to other nodes, the less it depends on other nodes, and the higher the closeness centrality the node has [24]. Relatively closeness centrality is expressed by equation (3), where $d_{i j}$ is the number of upper edges of the shortest distance between nodes $i$ and $j$.

$$
C_{R P i}^{-1}=\frac{\sum_{j=1}^{n} d_{i j}}{n-1} .
$$

(4) Eigenvector Centrality. Eigenvector centrality needs to use "factor analysis" to determine the "dimensions" of the distance between nodes. The corresponding position of each node in each dimension is called an "eigenvalue," and a series of such values are defined as eigenvectors [25]. For the network adjacency matrix, its element $a_{i j}$ means the status contribution of node $i$ to $j$. Let $x$ represent the eigenvector centrality, and the expression is shown in equation (4). That is, the centrality of a node is a function of the centrality of other nodes connected to this node

$$
x_{i}=a_{i 1} x_{1}+a_{i 2} x_{2}+\cdots+a_{i n} x_{n} .
$$

3.3.2. The Correlation between the Coreness and Centrality of Nodes. Coreness and centrality are both important indicators to determine the core nodes of the aviation network. The adjacency matrix A of the aviation network of "Belt and Road" was input into the UCINET software to calculate the coreness and 4 centrality indexes of all 522 nodes, as shown in Figure 2.

With the change in the coreness of nodes, the closeness centrality and betweenness centrality indexes of the nodes are evenly distributed. The index of Pearson correlation between the coreness, closeness centrality, and betweenness centrality of nodes is 0.428 and 0.427 , respectively, indicating that coreness has a weak correlation with closeness centrality and betweenness centrality; the Pearson correlation between the coreness, degree centrality, and eigenvector centrality is 0.783 and 0.861 , respectively, indicating that coreness has a strong positive correlation with degree centrality and eigenvector centrality; as the coreness of nodes increases, both the degree centrality and the eigenvector centrality show an increasing trend; the larger the coreness of a node, the higher the probability of high degree centrality and eigenvector centrality of the node. A node with small coreness has a relatively low-degree centrality and eigenvector centrality. 
TABLE 1: Statistics of node and edge extinction in each layer network.

\begin{tabular}{|c|c|c|c|c|c|c|}
\hline Coreness & Number of nodes & $\begin{array}{c}\text { The number of extinct } \\
\text { nodes }\end{array}$ & $\begin{array}{c}\text { The percentageof extinct } \\
\text { nodes }\end{array}$ & $\begin{array}{c}\text { The number } \\
\text { of edges }\end{array}$ & $\begin{array}{l}\text { The number } \\
\text { of extinct edges }\end{array}$ & $\begin{array}{c}\text { The percentage of } \\
\text { extinct edges }\end{array}$ \\
\hline 1 & 522 & 0 & $0.00 \%$ & 6822 & 0 & $0.00 \%$ \\
\hline 2 & 429 & 93 & $17.82 \%$ & 6642 & 180 & $2.64 \%$ \\
\hline 3 & 360 & 69 & $13.22 \%$ & 6402 & 240 & $3.52 \%$ \\
\hline 4 & 318 & 42 & $8.05 \%$ & 6182 & 220 & $3.22 \%$ \\
\hline 5 & 276 & 42 & $8.05 \%$ & 5886 & 296 & $4.34 \%$ \\
\hline 6 & 251 & 25 & $4.79 \%$ & 5656 & 230 & $3.37 \%$ \\
\hline 7 & 229 & 22 & $4.21 \%$ & 5417 & 239 & $3.50 \%$ \\
\hline 8 & 216 & 13 & $2.49 \%$ & 5249 & 168 & $2.46 \%$ \\
\hline 9 & 201 & 15 & $2.87 \%$ & 5036 & 213 & $3.12 \%$ \\
\hline 10 & 182 & 19 & $3.64 \%$ & 4727 & 309 & $4.53 \%$ \\
\hline 11 & 168 & 14 & $2.68 \%$ & 4471 & 256 & $3.75 \%$ \\
\hline 12 & 153 & 15 & $2.87 \%$ & 4175 & 296 & $4.34 \%$ \\
\hline 13 & 145 & 8 & $1.53 \%$ & 3996 & 179 & $2.62 \%$ \\
\hline 14 & 122 & 23 & $4.41 \%$ & 3444 & 552 & $8.09 \%$ \\
\hline 15 & 114 & 8 & $1.53 \%$ & 3242 & 202 & $2.96 \%$ \\
\hline 16 & 96 & 18 & $3.45 \%$ & 2731 & 511 & $7.49 \%$ \\
\hline 17 & 92 & 4 & $0.77 \%$ & 2607 & 124 & $1.82 \%$ \\
\hline 18 & 70 & 22 & $4.21 \%$ & 1901 & 706 & $10.35 \%$ \\
\hline 19 & 53 & 17 & $3.26 \%$ & 1363 & 538 & $7.89 \%$ \\
\hline
\end{tabular}

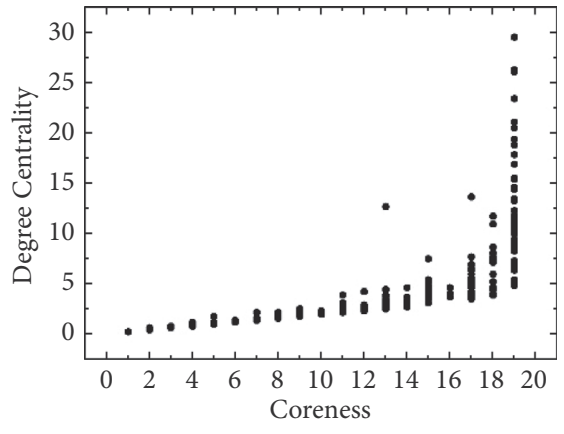

(a)

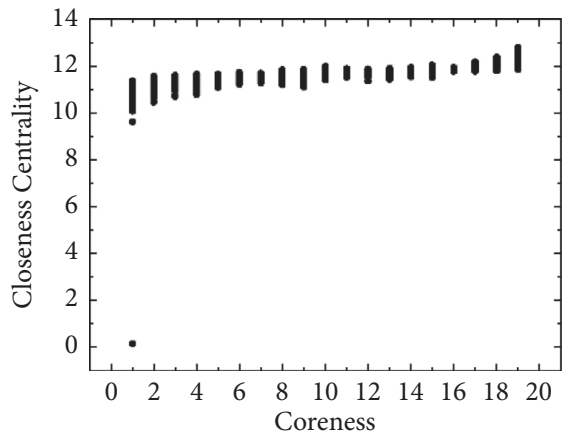

(b)

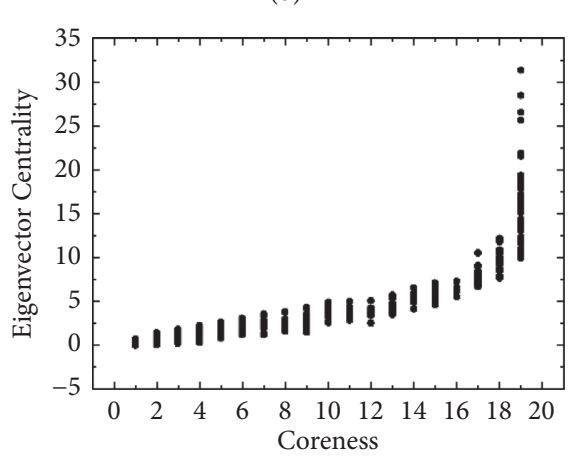

(d)

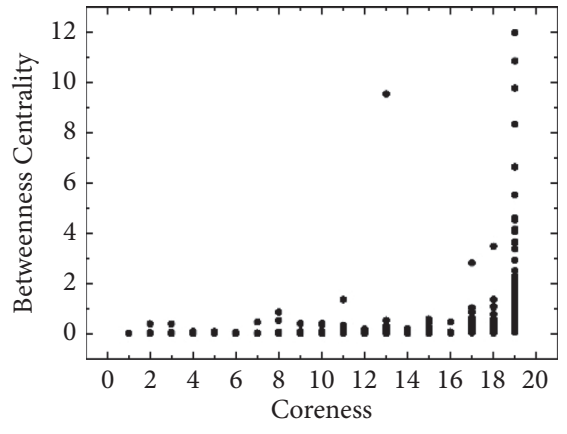

(c)

Figure 2: The coreness and centrality of nodes: (a) the coreness and degree centrality; (b) the coreness and closeness centrality; (c) the coreness and betweenness centrality; (d) the coreness and eigenvector centrality.

\section{Analysis of the $k$-Core Network Structure of the "Belt and Road" Aviation Network}

4.1. k-Core Networking of the "Belt and Road" Aviation Network. The "Belt and Road" aviation network can be divided into 19 levels by $k$-core decomposition. The navigation nodes at each layer and the air routes between them constitute 19 networks of different sizes, denoted as $k_{i}$ networks, where $i=1, \ldots, 19$; the structure of some networks is shown in Figure 3.

4.2. Comparative Analysis of the Properties of $k_{i}$ Network. The average distance, clustering coefficient, and degree distribution of the network are three important indicators to reveal the properties of the network. 


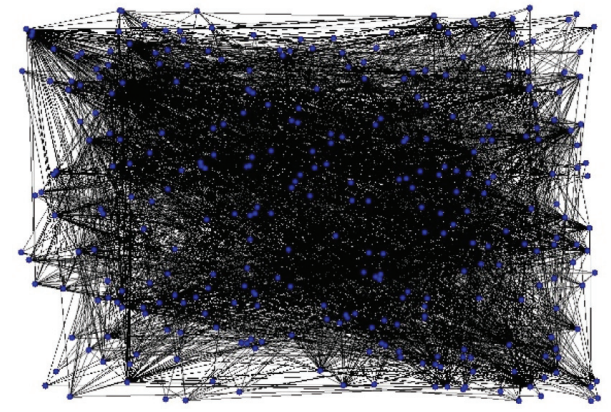

$K_{4}$ Network
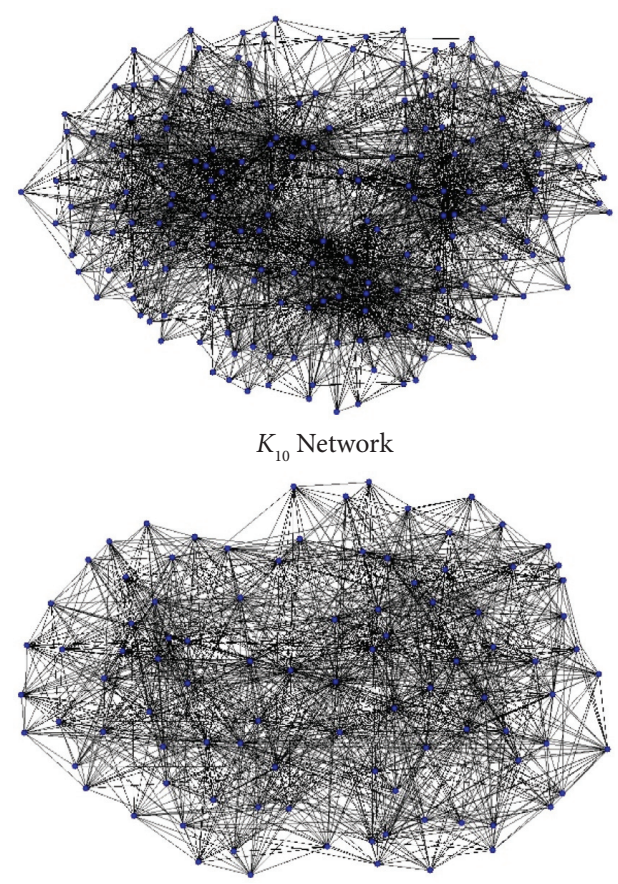

$K_{16}$ Network

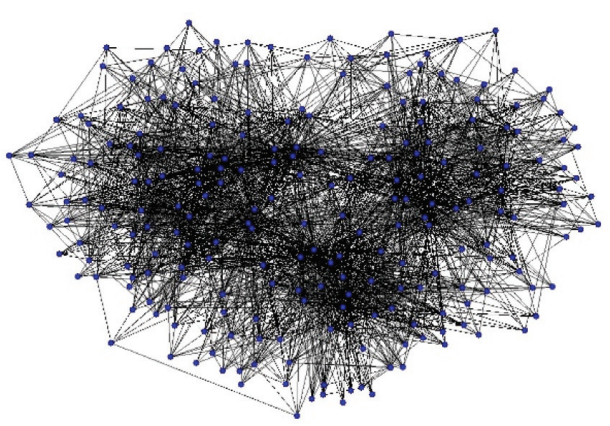

$K_{7}$ Network

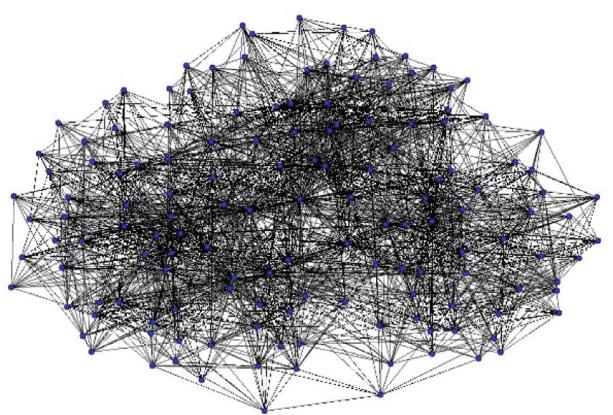

$K_{13}$ Network

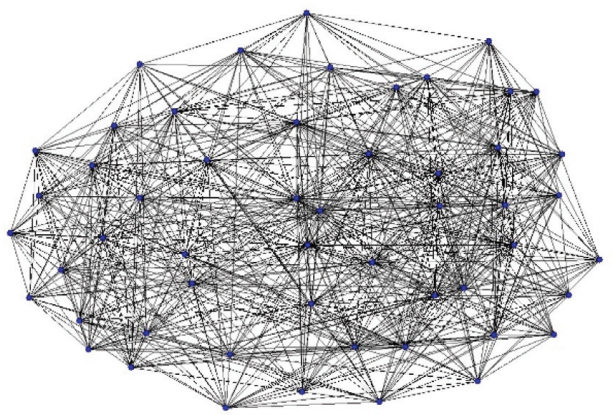

$K_{19}$ Network

FIgURE 3: Aviation network structure at different layers.

4.2.1. Average Distance. The distance of the network refers to the number of edges contained in the shortest path between any two nodes; the average distance is the mean of the distance between all pairs of nodes in the network, as shown in equation (5); the larger the value, the greater the network depth and the worse the convenience.

$$
L=\frac{\sum l_{i j}}{1 / 2 N(N+1)},
$$

where $N$ is the number of nodes and $l_{i j}$ is the number of edges with the shortest distance between nodes.

4.2.2. Clustering Coefficient. If a node $i$ has $d_{i}$ edges connected to other nodes, the clustering coefficient $C_{i}$ refers to the ratio of the number of edges that actually exist to the number of edges that may exist among $d_{i}$ nodes, as shown in equation (6). The clustering coefficient $C$ of the entire network is the mean of the clustering coefficient $C_{i}$ of all nodes $i$. The clustering coefficient is an indicator to reflect the degree of aggregation among nodes in the network.

$$
C_{i}=\frac{2 E_{i}}{d_{i}\left(d_{i}-1\right)}
$$

The 19 networks obtained from the $k$-core decomposition of the "Belt and Road" aviation network were input into the UCINET software to calculate the average distance and clustering coefficient of each network, and their values are displayed in Figure 4.

$\mathrm{t}$ can be seen from Figure 4 that the clustering coefficient of the network in each layer shows a gradual increase. The clustering coefficient of the $k_{1}$ network is the smallest, at 0.417 , and the clustering coefficient of the $k_{19}$ network is the largest, reaching 0.640; in general, the clustering coefficient of the network in each layer is relatively small compared with the clustering coefficient of China's aviation network and the global aviation network [26]; this reflects that the aggregation among the airports in the "Belt and Road" aviation network is relatively weak, and the status and role of hub 


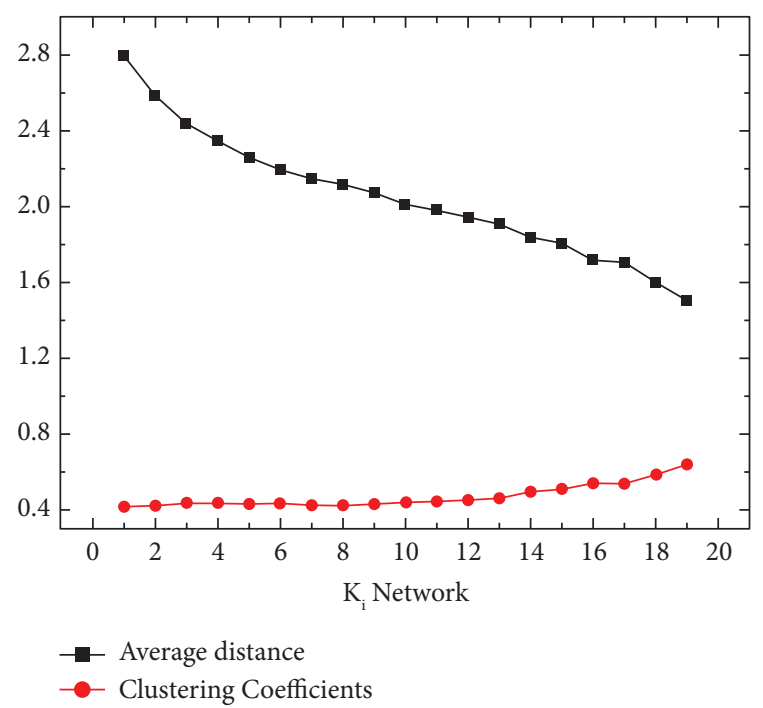

FIGURE 4: Average distance and clustering coefficient in different layer networks.

airports along the "Belt and Road" are not significant. The average distance of the network in each layer exhibits an obvious downward trend. The average distance of the $k_{1}$ network is the largest, at 2.796, which means two nodes in the $k_{1}$ network can reach each other after an average of 2.796 transfers; the average distance of the $k_{19}$ network is the smallest, at 1.505. The decrease in the average distance reflects that the degree of convenience of the network in each layer is continuously improved with the increasing degree of decomposition.

4.2.3. Degree Distribution. The degree distribution represents the probability that the degree value of a randomly selected node is exactly $k$. For the convenience of research, the cumulative degree distribution function is often used to reflect the degree distribution. It represents the probability distribution of nodes whose degree value is not less than $k$.

When the degree distribution conforms to the powerlaw distribution, the cumulative degree distribution function conforms to the power law with a power exponent of $\gamma-1$ [27], as shown in equation (7), where $\gamma$ is also called the degree distribution index. The smaller the value of $\gamma$, the greater the difference between the degree value of the nodes in the network and the stronger the scale-free property of the network. In the log-log coordinate system, the power-law distribution corresponds to a straight line [28].

$$
P(k)=\alpha \sum_{k^{\prime}=k}^{\infty} k^{\prime-\gamma}=\alpha k^{-(\gamma-1)} .
$$

Seven representative networks after $k$-core analysis were selected to calculate the node degree value and degree distribution function and the cumulative degree distribution curves in the log-log coordinate system, as shown in Figure 5.

In the log-log coordinate system, the cumulative degree distribution curve of each network corresponds

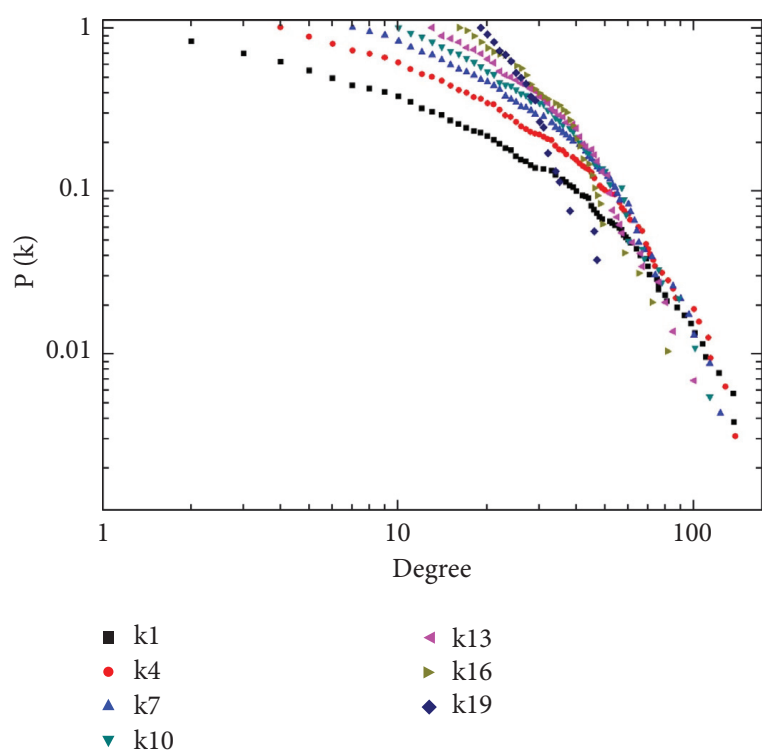

FIGURE 5: Cumulative degree distribution of different networks.

approximately to a straight line; thus, the above cumulative degree distribution curves conform to power-law distribution; fitting was performed on the above distribution curves, and the values of $a$ and $\gamma$ are shown in Table 2 .

It can be seen from Table 2 that as the value of $k$ increases, the degree distribution index $\gamma$ shows an increasing trend, indicating that with the increasing degree of network decomposition, the difference between the degree values of nodes in the network is continuously reduced, and the scale-free attribute of the network is constantly weakened. The value of $\gamma$ in $k_{1}$ and $k_{4}$ is less than 2 , revealing that there is a considerable proportion of nodes functioning as hubs in the network, and the hub nodes have a great impact on the network function; the value of $\gamma$ in the $k_{7}, k_{10}, k_{13}$, and $k_{16}$ networks is between 2 and 3 , and the network structure is similar to Internet router network and Movie actors network [29], suggesting that the connection between nodes in the network is not strong, and there are a small number of hub nodes, but the impact of hub nodes on network functions is further decreased. The value of $\gamma$ in the $k_{19}$ network exceeds 3 , which indicates that the network is similar to a random network, where the difference between the degree values of nodes in the network is not large. The realization of network functions does not depend on nodes with certain characteristics. Each node has a relatively limited influence on network functions, and the network is highly stable.

4.3. Analysis of the Connection of the Network in Each Layer. The analysis of the connection of the network in each layer can more in-depth reflect the law of connection between nodes and help to identify the core layer of the "Belt and Road" aviation network. Denote " $k$-residual set" as $k_{i}^{\prime}, i=1$, ..., 19. The connection of the network in each layer can be expressed by the interconnection between " $k$-residual sets"; the percentage of the number of interconnected edges 
TABLE 2: Fitting parameters of cumulative degree distribution curve.

\begin{tabular}{lccccccc}
\hline & $k_{1}$ & $k_{4}$ & $k_{7}$ & $k_{10}$ & $k_{13}$ & $k_{16}$ \\
\hline $\mathrm{A}$ & 1.194 & 3.623 & 8.187 & 16.335 & 38.632 & 123.747 & 4754.174 \\
$\Gamma$ & 1.609 & 1.835 & 2.007 & 2.169 & 2.394 & 2.702 & 3.852 \\
$R^{2}$ & 0.921 & 0.954 & 0.962 & 0.968 & 0.966 & 0.960 & 0.968 \\
\hline
\end{tabular}

between " $k$-residual sets" in the total number of edges of the nodes in the " $k$-residual sets" is displayed in Table 3.

Table 3 shows that the proportion of connections between the " $k$-residual set" of each layer and $k_{19}^{\prime}$ is the maximum, reflecting that $k_{19}^{\prime}$ is at the core; since the maximum coreness of the network is $19, k_{19}^{\prime}$ and $k_{19}$ are the same network. Therefore, it can be seen that the $k_{19}$ network has the highest percentage of connections with the networks in other layers.

\section{Research on the Levels of the "Belt and Road" Aviation Network}

5.1. Division of the "Belt and Road" Aviation Network. According to the structural parameters such as the number of nodes and edges in $k_{i}^{\prime}$, the largest connected subgraph, clustering coefficient, and network density, the categories of the levels of the network can be divided [30]. In $k_{i}^{\prime}$, since many nodes are isolated without connected to other nodes by edges, the nodes connected by edges in $k_{i}^{\prime}$ were regarded as "subgraphs," and the subgraph with the largest number of nodes is called the largest connected subgraph. The calculated structural parameters of the $k_{i}^{\prime}$ network are shown in Table 4.

Based on the method for network level division proposed [31] by Xiao et al. combining the variation characteristics of the largest connected subgraph, clustering coefficient, and network density in different cores, the "Belt and Road" aviation network was divided into three categories: core layer network, middle layer network, and detail layer network.

5.1.1. Core Layer Network. Since $k_{19}^{\prime}$ and $k_{19}$ are the same network, it can be regarded as the core network in the "Belt and Road" aviation network. Table 4 shows that this network has the largest clustering coefficient and network density, and 53 nodes in this network are connected to each other. Since coreness has a strong correlation with degree centrality and eigenvector centrality, the nodes of this layer have larger degree centrality and eigenvector centrality and play a greater role in connection. Since the network at this layer has a larger clustering coefficient and a smaller average distance, it exhibits stronger "small world" features and a higher degree of convenience; this network has the largest degree distribution index $\gamma$, indicating that there is little difference between node degree in the network, and the network has high stability. In addition, it can be seen from Table 3 that this network has the largest proportion of connections to networks at other layers, revealing that this network occupies a core position in the "Belt and Road" aviation network structure.
5.1.2. Middle Layer Network. The network composed of the nodes in $k_{13}^{\prime}, k_{14}^{\prime}, \ldots, k_{18}^{\prime}$ can be regarded as the middle layer network in the "Belt and Road" aviation network. Table 4 shows that in $k_{13}^{\prime}, k_{14}^{\prime}, \ldots, k_{18}^{\prime}$, the clustering coefficient and network density have increased significantly, and a larger connected subgraph appears in the network.

5.1.3. Detail Layer Network. It can be seen from Table 4 that in $k_{1}^{\prime}, k_{2}^{\prime}, \ldots, k_{12}^{\prime}$, not only the clustering coefficient is 0 but the network density is also small, and there are few nodes in the largest connected subgraph, reflecting that most of the nodes are in an isolated state. Therefore, the nodes in $k_{1}^{\prime}, k_{2}^{\prime}$, $\ldots, k_{12}^{\prime}$ constitute the detail layer network.

\subsection{Analysis of the Core Layer Network in the "Belt and Road" Aviation Network}

5.2.1. Composition of the Core Layer Network in the "Belt and Road" Aviation Network. The core network includes 53 airports in 37 countries; the country with the largest number of airports is the United Arab Emirates (5), followed by China and Saudi Arabia (4 each); the distribution is shown in Figure 6. The 37 countries covered by the core layer account for $56.92 \%$ of the total number of countries along the "Belt and Road," $86.45 \%$ of the GDP, and $93.74 \%$ of the total population along the "Belt and Road." They are highly representative.

5.2.2. Analysis of the Cohesive Subgroups of the Core Layer Aviation Network of "Belt and Road". Cohesive subgroup analysis can reveal the actual or potential interrelationship between nodes in the network. Cliques are the most closely related cohesive subgroups (Ren et al. 2000); in the network structure consisting of 53 airports in the core layer, although some airports are not prominent from a global perspective, they may play a crucial role in the connection of the core layer; therefore, identifying cliques based on the strength of the connection between nodes is of great significance for effectively enhancing the overall connection of the aviation network.

UCINET software was used to analyze cliques in the core layer network. With a minimum of 3 nodes as the standard, a total of 857 cliques were identified, of which the largest clique contains 13 nodes and the smallest clique contains 4 nodes. Through the analysis of the identified cliques, the following characteristics of the nodes in the core layer network were discovered:

(1) Although there are many cliques identified, a large number of "overlapping" nodes exist; for example, Qatar's Doha (QA-DOH) appears in 582 cliques 


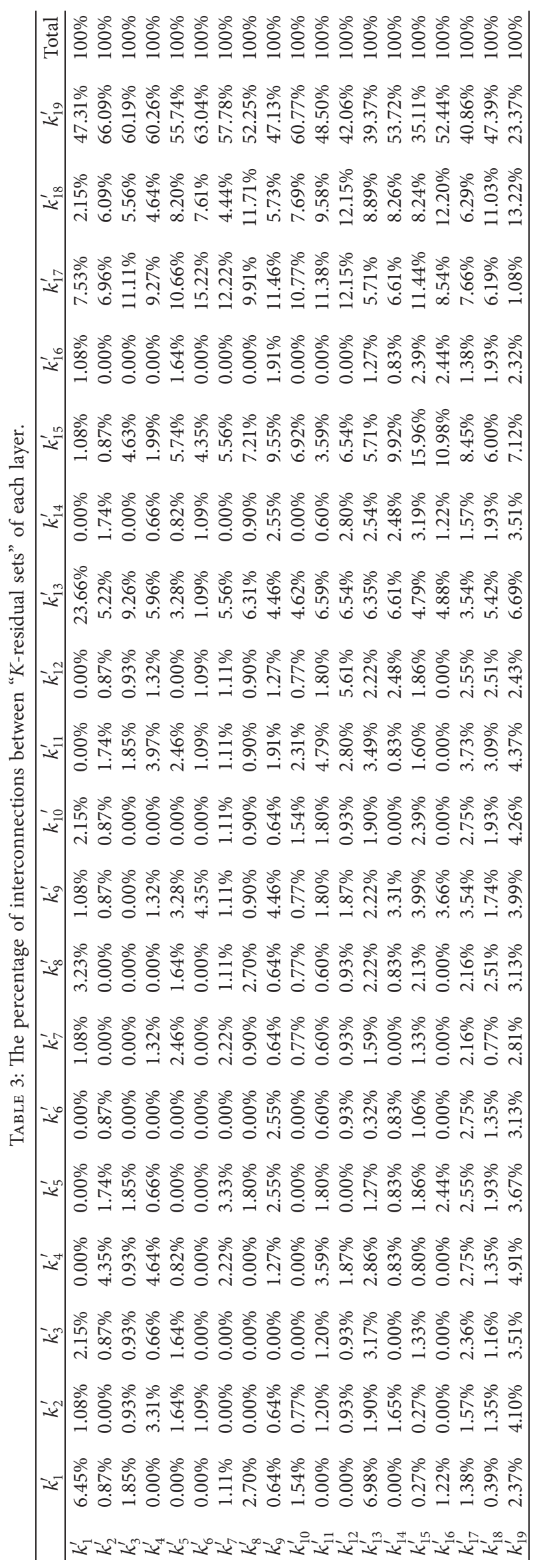


TABLE 4: Structure parameters of the network at each layer.

\begin{tabular}{|c|c|c|c|c|c|}
\hline The number of nodes & The number of edges & $\begin{array}{l}\text { The largest conr } \\
\text { The number of nodes }\end{array}$ & $\begin{array}{l}\text { hected subgraph } \\
\text { The number of edges }\end{array}$ & Network density & Clustering coefficient \\
\hline 93 & 6 & 2 & 1 & 0.0007 & 0.0000 \\
\hline 69 & 0 & 1 & 0 & 0.0000 & 0.0000 \\
\hline 42 & 1 & 2 & 1 & 0.0006 & 0.0000 \\
\hline 42 & 7 & 3 & 2 & 0.0041 & 0.0000 \\
\hline 25 & 0 & 1 & 0 & 0.0000 & 0.0000 \\
\hline 22 & 0 & 1 & 0 & 0.0000 & 0.0000 \\
\hline 13 & 2 & 2 & 1 & 0.0128 & 0.0000 \\
\hline 15 & 3 & 2 & 1 & 0.0143 & 0.0000 \\
\hline 19 & 8 & 2 & 1 & 0.0234 & 0.0000 \\
\hline 14 & 2 & 2 & 1 & 0.0110 & 0.0000 \\
\hline 15 & 8 & 4 & 4 & 0.0381 & 0.0000 \\
\hline 8 & 6 & 3 & 2 & 0.1071 & 0.0000 \\
\hline 23 & 29 & 11 & 24 & 0.0573 & 0.4240 \\
\hline 8 & 2 & 2 & 1 & 0.0536 & 0.0000 \\
\hline 18 & 57 & 12 & 55 & 0.1863 & 0.2070 \\
\hline 4 & 1 & 2 & 1 & 0.1667 & 0.0000 \\
\hline 22 & 63 & 17 & 58 & 0.1245 & 0.4740 \\
\hline 17 & 57 & 14 & 57 & 0.2375 & 0.3650 \\
\hline 53 & 1363 & 53 & 1363 & 0.4946 & 0.6400 \\
\hline
\end{tabular}

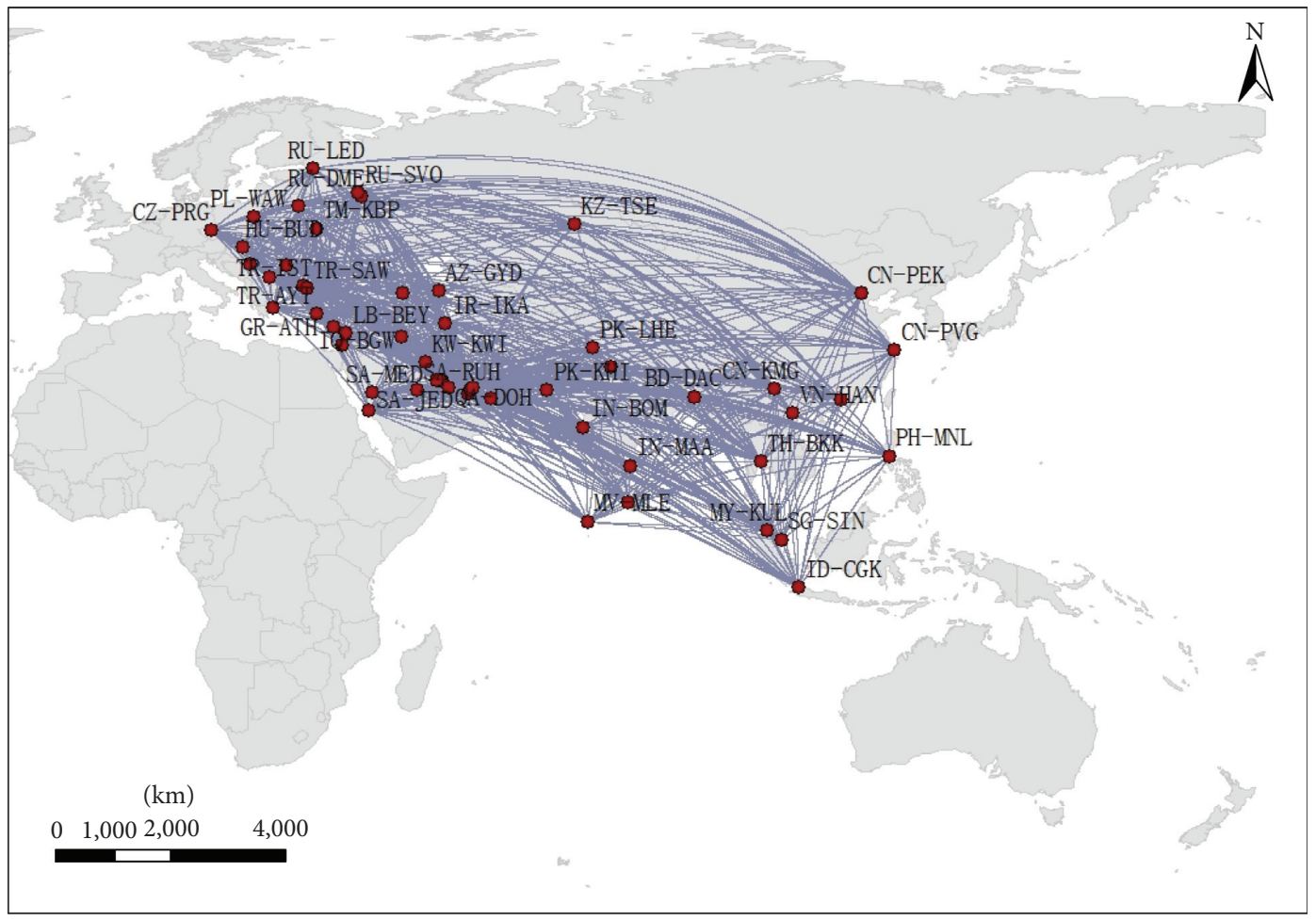

FIGURE 6: Core layer aviation network.

and is the node overlapped most. In addition, airports that "overlap" frequently include Turkey's Chistanbul (TR-IST), UAE's Dubai (AE-DXB), and Thailand's Bangkok (AE-DXB), which appear in 560, 374, and 371 cliques, respectively; this indicates that these airports play an important role in the connection of the core layer network, and they are also the most important hub airports in the "Belt and Road" aviation network.
China's 4 airports such as Beijing Capital (CN-PEK), Shanghai Pudong (CN-PVG), Guangzhou (CN-CAN), and Kunming (CN-KMG) appear in 183, 67, 41, and 28 cliques, respectively; except for Beijing, the number of cliques where the other three airports are engaged is much lower than the average value of 135 , indicating that their status in the core layer is not prominent. 


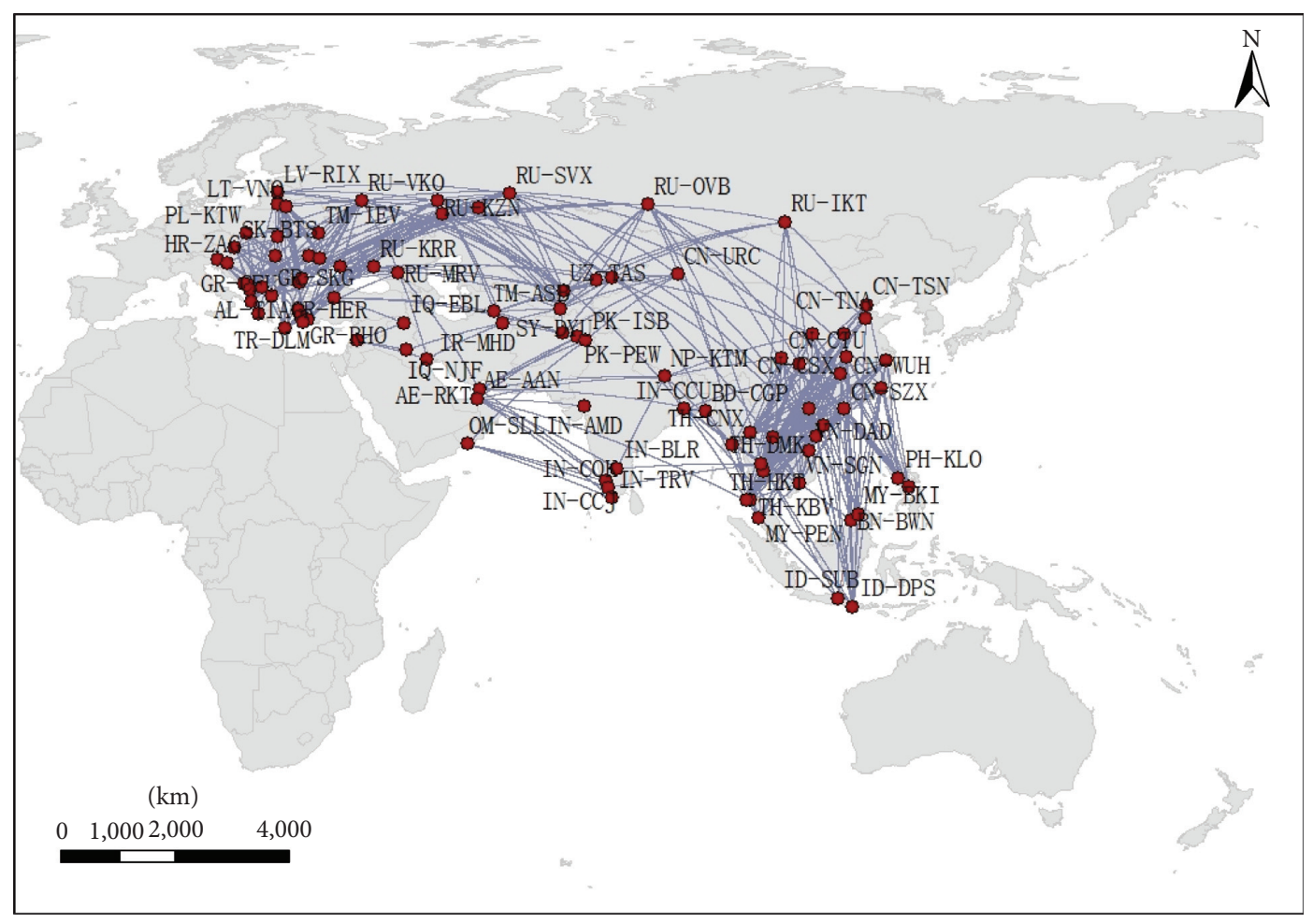

FIGURE 7: Middle layer network, aviation network.

(2) The "Belt and Road" route can be divided into five major sectors: East Asia, Southeast Asia, Central Asia, the Middle East, and Europe. By analyzing the composition of airports in cliques, it can be seen that there exists a relatively close connection between the airports within each major regional sector, and a large number of clique members are from the same sector; the analysis of some large cliques showed that the four sectors such as East Asia, Southeast Asia, Central Asia, and the Middle East are closely related, and the airports in these four sectors often appear in the same clique. Europe only has a close connection with the Middle East sector, while its connection with the three sectors such as East Asia, Southeast Asia, and Central Asia is weak.

(3) Factors such as economy, population, and land do not have a significant impact on the status of airports in the network. Airports in China, India, Russia, and other countries do not have many cliques, while airports in Qatar, Turkey, UAE, and Thailand have a large number of cliques due to their unique geographical location, showing that geographical location is currently the main factor determining the status of the nodes in "Belt and Road" aviation network.

5.3. Analysis of the Middle Layer Network in the "Belt and Road" Aviation Network. The middle layer network includes 92 airports in 39 countries; the country with the largest number of airports is China (15), followed by Russia (9) and India (6 each); the distribution is shown in Figure 7.
The middle layer network contains 92 airports, among which the airports with high degree are Moscow Vnukovo International Airport (RU-VKO), Bangkok Don Mueang International Airport (TH-DMK), Indonesia Denpasar International Airport (ID-DPS), and Tan Son Nhat International Airport (VN-SGN) in Vietnam. Airports in the middle layer network have a high $K$-core value. These airports are mostly secondary aviation hubs in the country where they are located, and they have a certain degree of influence in the network. Compared with the airports in the core layer network, the airports in the middle layer network are connected to relatively single objects by air routes, and the clustering coefficient is not high, suggesting that these airports do not tend to form clusters.

\subsection{Analysis of the Detail Layer Network in the "Belt and Road"} Aviation Network. The detail layer network consists of 460 airports in 64 countries; the distribution is shown in Figure 8 . Despite a large number of nodes, this network is the most sparse, with only 221 routes, accounting for $3.24 \%$ of the total number of routes of the "Belt and Road" aviation network. Among them, 258 airports have no connection with other airports at the same layer by air routes; this shows that they are on the outer edge of the "Belt and Road" aviation network, and there are a large number of isolated nodes or plenty of relatively independent subgroups formed by a large quantity of low-degree nodes.

The node airports in the detail layer network only exist in the "Belt and Road" aviation network by connecting with the airports in the core layer network or the middle layer network. Therefore, they are the radiation and expansion of 


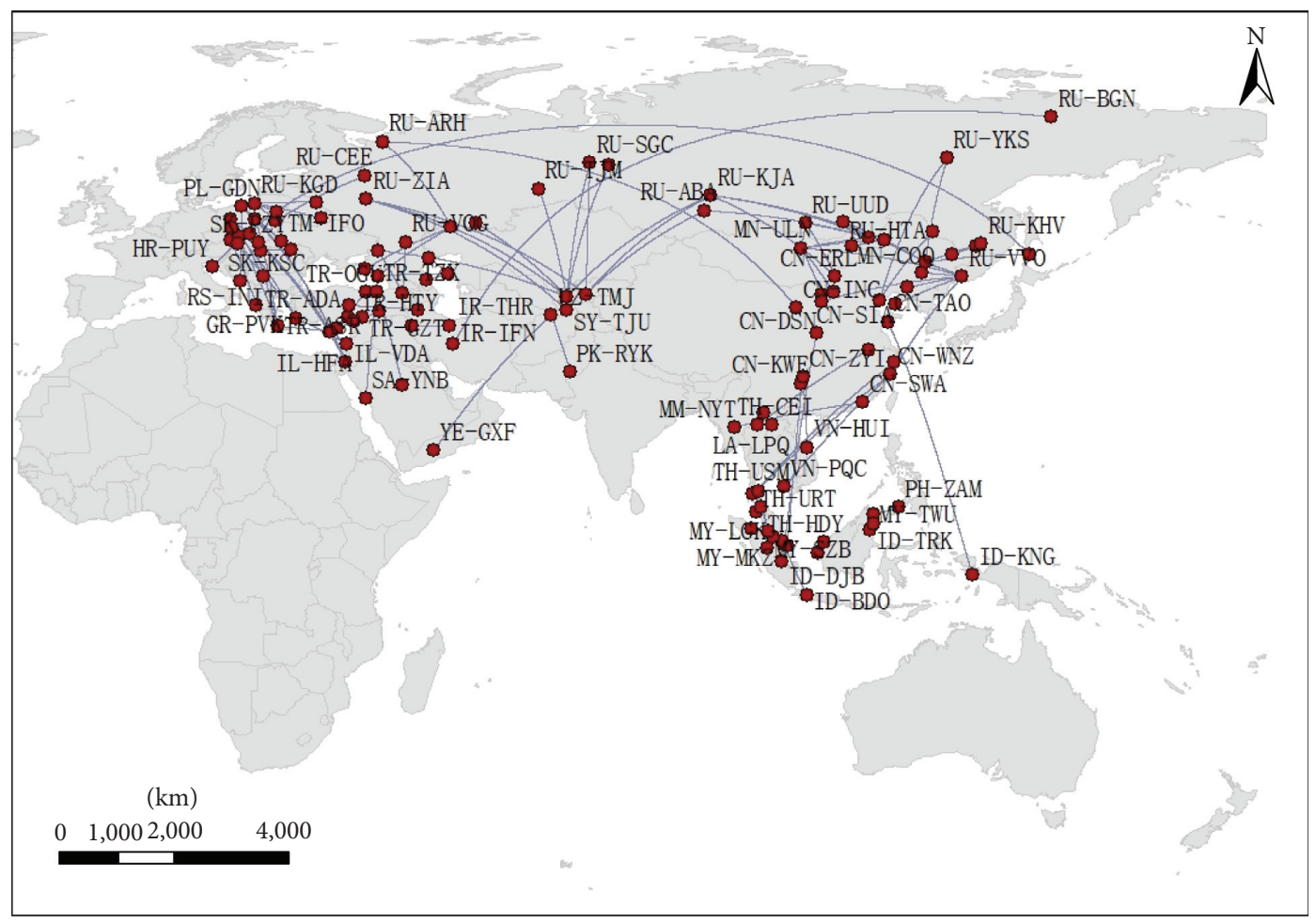

FIGURE 8: Detail layer network, aviation network.

the core layer network and the middle layer network to the periphery. Although the airports in the network at this layer play a small role, they effectively increase the transportation service coverage of the entire aviation network and are also important components of the "Belt and Road" aviation network.

\section{Conclusion}

In this paper, the data on airports and air routes were collected to construct the "Belt and Road" aviation network model; $k$-core decomposition was conducted, and a network structure model with 19 levels was obtained; the relationship between the coreness and centrality of nodes in the network was studied, the attribute variation of the network at different levels was analyzed, and the connection between these networks was explored; finally, based on the structural characteristics of the 19-layer network, the "Belt and Road" aviation network was divided into three categories: the core layer, the middle layer, and the detail layer networks, and their respective characteristics were investigated.

The following conclusions were obtained in this research:

(1) The $k$-core analysis based on the "degree" value can distinguish different cohesive groups in the aviation network hierarchically, to realize a hierarchical network; through the $k$-core decomposition, the group size is reduced from the outside to the inside, and finally a relatively important core layer is obtained, which can effectively reduce the complexity of the aviation network.
(2) The highest-level network obtained by $k$-core decomposition can be used as the core layer of the "Belt and Road" aviation network, which includes 53 general aviation airports in 37 countries and shows strong "small world" characteristics; the difference between the degree values of nodes is not large, suggesting that the network has high stability.

(3) In the future, the connection between Europe and East Asia, Southeast Asia, and Central Asia should be strengthened, and the density of air routes should be increased; geographical factors are the dominant factors determining the status of the airport, indicating that air transport mainly plays a role in stimulating personnel exchange along the "Belt and Road," and its role in economic and trade development needs to be further intensified.

\section{Data Availability}

The data used to support the findings of this study are available from the corresponding author upon request.

\section{Conflicts of Interest}

The authors declare that they have no conflicts of interest.

\section{Acknowledgments}

This study was supported by National Social Science Foundation of China (16BGL014). 


\section{References}

[1] S. Wandelt and X. Sun, "Evolution of the international air transportation country network from 2002 to 2013," Transportation Research Part E, vol. 82, p. 101034, 2015.

[2] K. Y. Tommy, C. Collin, W. H. Wong, and A. Zhang, "The evolution of aviation network: global airport connectivity index 2006-2016," Transportation Research Part E: Logistics and Transportation Review, vol. 133, p. 101826, 2020.

[3] L. Hong-Kun and T. Zhou, "Empirical study of Chinese city airline network," Acta Physica Sinica, vol. 56, no. 1, pp. 106-112, 2007.

[4] Z. Xiao-zhou, K.-S. Jiang, and K. Cheng, "Comparative analysis on the centralized level of hub airport of Chinese aviation network," Systems Engineering, vol. 28, pp. 39-45, 2010.

[5] F. Du, J. Wang, J. Xie, and D. Du, "Spatial pattern and change of China's international air transport network since the Belt and Road Initiative," Progress in Geography, vol. 38, pp. 963-972, 2019.

[6] J. Wang, H. Wang, and J. Jiao, "China's international aviation transport to the Belt and Road Initiative area," Progress in Geography, vol. 34, pp. 554-562, 2015.

[7] H. Yao, "Multi-resolution wavelet decomposition of Chinese aviation network," Journal of Southwest Jiaotong University, vol. 48, pp. 141-146, 2013.

[8] H.-J. Wang and M. I. A. O. Chang-hong, "Network structure and regional difference of aviation links in China," Scientia Geographica Sinica, vol. 35, pp. 1220-1229, 2015.

[9] J. Jiao and J. Wang, "Spatial structure and evolution of Hainan Airlines Network: an analysis of complex network," Geographical Research, vol. 33, pp. 926-936, 2014.

[10] B. Leng, Y. Yang, Y. Li, and S. Zhao, "Spatial characteristics and complex analysis: a perspective from basic activities of urban networks in China," Acta Geographica Sinica, vol. 66, pp. 199-211, 2011.

[11] K. Sheng, Y. Yang, and H. Zhang, "Cohesive subgroups and underlying factors in the urban network in China," Geographical Research, vol. 38, pp. 2639-2652, 2019.

[12] W. Liu, M. Han, and Z. Xie, "Connectivity characteristics and communities identification of world city network based on global airline," Economic Geography, vol. 40, pp. 34-40, 2020.

[13] S. P. Borgatti and M. G. Everett, "Models of core-periphery structures," Social Networks, vol. 21, pp. 375-395, 1999.

[14] M. E. J. Newman, "The structure of scientific collaboration networks," Proceedings of the National Academy of Sciences, vol. 98, no. 2, pp. 404-409, 2001.

[15] M. Kitsak, L. K. Gallos, S. Havlin et al., "Identification of influential spreaders in complex networks," Nature Physics, vol. 6, no. 11, pp. 888-893, 2010.

[16] Y. He and J. Tan, "Study on SINA micro-blog personalized recommendation based on semantic network," Expert Systems with Applications, vol. 42, no. 10, pp. 4797-4804, 2015.

[17] S. B. Seidman, "Network structure and minimum degree," Social Networks, vol. 5, no. 3, pp. 269-287, 1983.

[18] R. Oliver, "The $k$-core and branching processes," Combinatorics, Probability \& Computing, vol. 17, pp. 111-136, 2008.

[19] M. Zhang, S. Ge, Y. Jia, and N. Wang, "Analysis of cohesive characteristics in scientific collaboration networks based on k-core," Systems Engineering-Theory \& Practice, vol. 40, pp. 1821-1831, 2020.

[20] L. Huang, Y.-D. Zhao, and L. Mei, "Research on multi-sided market competition of social media personal data resource - an empirical analysis based on $k$-core network," Soft Science, vol. 33, pp. 1-7, 2019.

[21] Z.-M. Ren, J.-G. Liu, F. Shao, Z.-L. Hu, and Q. Guo, "Analysis of the spreading influence of the nodes with minimum $K$-shell value in complex networks," Acta Physica Sinica, vol. 62, no. 10, Article ID 108902, 2013.

[22] Y. Zhang, A. Zhang, Z. Zhu, and K. Wang, "Connectivity at Chinese airports: the evolution and drivers," Transportation Research Part A: Policy and Practice, vol. 103, pp. 490-508, 2017.

[23] A. L. Barabási and R. Albert, "Emergence of scaling in random network," Science, vol. 286, pp. 509-512, 1999.

[24] S. Abe and N. Suzuki, "Scale-free network of earthquakes," Europhysics Letters, vol. 65, no. 4, pp. 581-586, 2004.

[25] R. Albert and A.-L. Barabási, "Statistical mechanics of complex networks," Reviews of Modern Physics, vol. 74, no. 1, pp. 47-97, 2002.

[26] J. Zhang, X.-B. Cao, W.-B. Du, and K.-Q. Cai, "Evolution of Chinese airport network," Physica A: Statistical Mechanics and Its Applications, vol. 389, no. 18, pp. 3922-3931, 2010.

[27] M. E. J. Newman, "The structure and function of complex networks," SIAM Review, vol. 45, no. 2, pp. 167-256, 2003.

[28] P. L. Krapivsky, S. Redner, and F. Leyvraz, "Connectivity of growing random networks," Physical Review Letters, vol. 85, no. 21, pp. 4629-4632, 2000.

[29] Z. Kou and C. Zhang, "Reply networks on bulletin board system," Physical Review E-Statistical Physics, Plasmas, Fluids, and Related Interdisciplinary Topics, vol. 67, p. 36117, 2003.

[30] S. Carmi, S. Havlin, S. Kirkpatrick, Y. Shavitt, and E. Shir, "A model of internet topology using $k$-shell decomposition," Proceedings of the National Academy of Sciences, vol. 104, no. 27, pp. 11150-11154, 2007.

[31] L. Xiao, G. Chen, J. Sun, S. Han, and C. Zhang, "Exploring the topic hierarchy of digital library research in China using keyword networks: a K-core decomposition approach," Scientometrics, vol. 108, no. 3, pp. 1085-1101, 2016. 